\title{
ON FUNCTIONS OF BOUNDED BOUNDARY ROTATION
}

\section{MING-CHIT LIU}

Abstract. Let $U=\left\{z=r e^{i \theta} \mid r<1\right\}$. For $k \geqq 2$ let $V_{k}$ be the class of normalized analytic functions $f(z)$ such that the boundary rotation of $f(U)$ is at most $k \pi$. Let $A(r)$ be the integral

$$
\int_{0}^{2 \pi} \int_{0}^{r}\left|f^{\prime}\left(\rho e^{i \theta}\right)\right|^{2} \rho d \rho d \theta
$$

$L(r)$ the length of the image of the circle $|z|=r$ under the mapping $f(z)$. In this paper the author proves that for $z \in U$ if $f(z) \in V_{k}$ then

$$
\limsup _{r \rightarrow 1}\left(\operatorname{Sup}_{f \in V_{k}} L(r)\right)\left(\pi A(r) \log \left(\frac{1+r}{1-r}\right)\right)^{-1 / 2} \leqq k .
$$

This generalizes to arbitrary $k \geqq 2$ the recent result of Nunokawa for the case $k=2$.

Let $U$ be the unit disk, $|z|<1$. For fixed $k \geqq 2$, by $V_{k}$ we denote the class of functions $f(z)$ satisfying the following conditions:

(i) $f(z)$ is analytic and $f^{\prime}(z) \neq 0$ for $z \in U$.

(ii) $f(z)$ is normalized by the requirements $f(0)=0$ and $f^{\prime}(0)=1$.

(iii) The boundary rotation of $f(U)$ is at most $k \pi$, that is

$$
\int_{0}^{2 \pi}\left|\operatorname{Re}\left\{1+\frac{z f^{\prime \prime}(z)}{f^{\prime}(z)}\right\}\right| d \theta \leqq k \pi
$$

where $r e^{i \theta}=z \in U$.

The concept of bounded boundary rotation originates from Löwner [1]. Extensive investigations are due to Paatero [3].

Let $K$ denote the class of functions $f(z)=z+\sum_{n=2}^{\infty} a_{n} z^{n}$, convex in $U$, i.e. $\operatorname{Re}\left\{1+z f^{\prime \prime}(z) / f^{\prime}(z)\right\}>0$. It is clear that $V_{2}=K$.

Let $C(r)$ denote the image of the circle $|z|=r<1$ under some mapping $f(z), L(r)$ the length of $C(r)$. By $A(r)$ we denote the integral

$$
\int_{0}^{2 \pi} \int_{0}^{r}\left|f^{\prime}(t)\right|^{2} \rho d \rho d \theta \quad\left(t=\rho e^{i \theta}\right)
$$

which is the generalized area of the image of the set $|z| \leqq r$ under the mapping $f(z)$.

Recently, Nunokawa [2, p. 332] obtained:

Received by the editors September 15, 1970.

AMS 1970 subject classifications. Primary 30A32; Secondary 30A04.

Key words and phrases. Analytic mapping, function of bounded boundary rotation, convex function, curve length, order of infinity.

Copyright (c) 1971, American Mathematical Society 
THEOREM N. If $f(z) \in K\left(=V_{2}\right)$ then

$$
L(r)=O\left(A(r) \log \frac{1}{1-r}\right)^{1 / 2},
$$

as $r \rightarrow 1$.

In this paper we shall extend Theorem $\mathrm{N}$ to all classes $V_{k}$ by proving

THEOREM. Let $2 \leqq k<\infty$. If $f(z) \in V_{k}$, then

$$
\limsup _{r \rightarrow 1}\left(\operatorname{Sup}_{f \in V_{k}} L(r)\right)\left(\pi A(r) \log \left(\frac{1+r}{1-r}\right)\right)^{-1 / 2} \leqq k .
$$

Proof. Let $a$ be a fixed constant, $0<a<r=|z|<1$. Nunokawa $\left[2\right.$, p. 333] has shown that if $f(z)$ has a nonvanishing derivative $f^{\prime}(z)$ in $U$ then

$$
L(r)=\int_{0}^{2 \pi}\left|z f^{\prime}(z)\right| d \theta \leqq C+(A(r) J / a)^{1 / 2} \quad\left(z=r e^{i \theta}\right),
$$

where

$$
C=\int_{0}^{a} \int_{0}^{2 \pi}\left|t f^{\prime \prime}(t)+f^{\prime}(t)\right| d \theta d \rho \quad\left(t=\rho e^{i \theta}\right)
$$

and

$$
J=\int_{0}^{r} \int_{0}^{2 \pi}\left|1+\frac{t f^{\prime \prime}(t)}{f^{\prime}(t)}\right|^{2} d \theta d \rho .
$$

We observe that since the functions $f(z)$ of the class $V_{k}$ comprise a normal family there exists a constant $A=A(a, k)$ depending only upon $a$ and $k$ such that $C \leqq A$. An estimate for $J$ for the class $V_{k}$ follows from the following result due to Robertson [4, p. 1480]:

$$
\begin{aligned}
\int_{0}^{2 \pi}\left|1+t \frac{f^{\prime \prime}(t)}{f^{\prime}(t)}\right|^{2} d \theta & =2 \pi+\rho^{2} \int_{0}^{2 \pi}\left|\frac{f^{\prime \prime}(t)}{f^{\prime}(t)}\right|^{2} d \theta \\
& \leqq 2 \pi\left(1+\frac{k^{2} \rho^{2}}{\left(1-\rho^{2}\right)}\right)<\frac{2 \pi k^{2}}{1-\rho^{2}} .
\end{aligned}
$$

Then

$$
J<2 \pi k^{2} \int_{0}^{r} \frac{d \rho}{1-\rho^{2}}=\pi k^{2} \log \left(\frac{1+r}{1-r}\right) .
$$


Since $\int_{0}^{2 \pi}\left|f^{\prime}(z)\right|^{2} d \theta \geqq 2 \pi$ it follows that $A(r) \log ((1+r) /(1-r))$ is unbounded as $r \rightarrow 1$. Since $a$ can be chosen arbitrarily close to one the inequality (3) follows at once.

REMARK. It is interesting to note that recently, M. S. Robertson [5, p. 97] has considered a family of functions that includes the class $K$ as a proper subfamily. For $-\frac{1}{2} \pi<\alpha<\frac{1}{2} \pi$, we say that $f(z) \in S_{\alpha}$ if

(i) $f(z)$ is analytic and $f^{\prime}(z) \neq 0$ for $z \in U$.

(ii) $f(0)=0$ and $f^{\prime}(0)=1$.

(iii) For $z \in U$,

$$
\operatorname{Re}\left\{e^{i \alpha}\left(1+\frac{z f^{\prime \prime}(z)}{f^{\prime}(z)}\right)\right\}>0 .
$$

Clearly, $S_{0}=K$. We can extend inequality (2) from the class $K$ to all classes $S_{\alpha}\left(|\alpha|<\frac{1}{2} \pi\right)$ as follows:

For $f(z) \in S_{\alpha}\left(|\alpha|<\frac{1}{2} \pi\right)$ we have

$$
\lim \sup \left(\operatorname{Sup}_{r \in S_{\alpha}} L(r)\right)\left(\pi A(r) \log \left(\frac{1+r}{1-r}\right)\right)^{-1 / 2} \leqq 2 \cos \alpha .
$$

The proof of (4) is similar to that in our Theorem. Let $f(z) \in S_{\alpha}$. Then there is a function $P(z)$ with positive real part and regular in $U$, $P(0)=1,\left|P^{(n)}(0)\right| \leqq 2(n !), n=1,2,3, \cdots$, such that

$$
e^{i \alpha}\left(1+\frac{z f^{\prime \prime}(z)}{f^{\prime}(z)}\right)=P(z) \cos \alpha+i \sin \alpha .
$$

Then

$$
\begin{aligned}
\int_{0}^{2 \pi}\left|1+\frac{z f^{\prime \prime}(z)}{f^{\prime}(z)}\right|^{2} d \theta & =2 \pi+\int_{0}^{2 \pi}\left|\frac{z f^{\prime \prime}(z)}{f^{\prime}(z)}\right|^{2} d \theta \\
& =2 \pi+\int_{0}^{2 \pi}|(P(z)-1) \cos \alpha|^{2} d \theta \\
& \leqq 2 \pi+\frac{8 \pi r^{2} \cos ^{2} \alpha}{1-r^{2}}=\frac{8 \pi \cos ^{2} \alpha}{1-r^{2}}(1+o(1))
\end{aligned}
$$

as $r \rightarrow 1$. Then (4) follows.

My thanks are due to the referee for some useful suggestions shortening the original proofs.

\section{REFERENCES}

1. K. Löwner, Untersuchungen über die Verzerrung bei konformen Abbildungen des Einheitskreises $|z|<1$, die durch Funktionen mit nicht verschwindender Ableitung geliefert werden, Ber. Kön. Sächs. Ges. Wiss. Leipzig 69 (1917), 89-106. 
2. M. Nunokawa, A note on convex and Bazilevič functions, Proc. Amer. Math. Soc. 24 (1970), 332-335. MR 40 \#437.

3. V. Paatero, Über die konforme Abbildung von Gebieten deren Ränder von beschränkter Drehung sind, Ann. Acad. Sci. Fenn. Ser. A 33 (1931), 77 pp.

4. M. S. Robertson, Coefficients of functions with bounded boundary rotation. Canad. J. Math. 21 (1969), 1477-1482.

5. - Univalent functions $f(z)$ for which $z f^{\prime}(z)$ is spirallike, Michigan Math. J. 16 (1969), 97-101. MR 39 \#5785.

University of Hong Kong, Hong Kong 